\title{
Interferometric study of piezoelectric degradation in ferroelectric thin films
}

\author{
A. Kholkin, E. Colla, K. Brooks, P. Muralt, M. Kohli, T. Maeder, D. Taylor, and N. Setter \\ Laboratoire de Céramique, Ecole Polytechnique Fédérale de Lausanne \\ CH-1015 Lausanne, Switzerland
}

Version of record: Microelectronic Engineering 29 (1-4), 261-264, 1995

http://hdl.handle.net/10.1016/0167-9317(95)00157-3

\begin{abstract}
Degradation of piezoelectric properties has been studied in ferroelectric PZT films by means of optical interferometry. The degradation under dc bias and aging of poled films have been observed. The decay of piezoelectric coefficient with time is described by a logarithmic law with aging rates comparable to those of switching polarization. The aging rates are shown to be sensitive to poling conditions and to orientation of the poling field with respect to the direction of preferred polarization. The fatigue measurements revealed a decrease of piezoelectric coefficient with a simultaneous shift of piezoelectric hysteresis loops. Degradation tests on thin membranes covered by PZT films have been reported.
\end{abstract}

\section{Introduction}

Ferroelectric thin films are very attractive for use in rnicroelectromechanical systems (MEMS) because of their superior piezoelectric properties as compared to conventional $\mathrm{ZnO}$ films. Recently, several microelectromechanical devices based on ferroelectric thin films have been demonstrated such as ultrasonic piezoelectric motors, pressure sensors, cantilever beams etc. (see e.g. [1-3]). However, very little is known about the degradation of piezoelectric properties with time which will determine the long-term behavior of ferroelectric films in MEMS. It is expected that piezoelectric degradation in thin films will be different from that of bulk ceramics because of their essential two-dimensional geometry, additional stresses induced during the film deposition, and different microstructure.

In this paper, an investigation of degradation behavior of the piezoelectric properties of sol-gel derived PZT films is reported for the first time. The degradation tests on piezoelectric thin film devices are also presented.

\section{Experimental}

$\mathrm{PbZr}_{x} \mathrm{Ti}_{1-x} \mathrm{O}_{3}$ (PZT) thin films were prepared on $\mathrm{Si} / \mathrm{SiO}_{2} / \mathrm{Ti} / \mathrm{Pt}$ substrates by sol-gel technique using a standard method described by Budd [4]. The film microstructures were columnar and mostly (111) textured. The composition was near the morphotropic phase boundary $(\mathrm{x}=0.45)$ and the thickness of the films was about 0.2-0.3 $\mu \mathrm{m}$. A detailed description of the sol-gel process can be found elsewhere [5]. The sputter and sol-gel techniques were applied for the deposition of the PZT films on Si and Zr membranes with thicknesses from 4 to $20 \mu \mathrm{m}$ [3].

A sensitive laser interferometer with a resolution of about $2 \cdot 10^{-4} \mathrm{~nm}$ was used to measure the small fieldinduced displacements. For the measurements of longitudinal piezoelectric coefficients a double-beam optical scheme [6] was utilized in which the laser beam probed both the top electrode and the back side of the Si wafer. Thus the movement of the PZT film with Si substrate as a whole was excluded. A single-beam optical arrangement [7] was used to measure the displacements of thin membranes covered by PZT films. 


\section{Results and discussion}

\subsection{Aging}

Aging is defined as a spontaneous change of thin film properties after cooling from above the Curie point or after electrical pretreatment to reach the deaged initial state. Because the heating of the films is impossible inside the interferometer an electrical pretreatment which consisted of exposing the film to an ac field of magnitude exceeding the coercive one was used. Following this, the film was poled with the voltage pulses of different amplitudes and durations and the piezoelectric coefficient was monitored for several hours. A typical piezoelectric response under dc bias and after poling is shown in Fig. 1. Both degradation under dc bias and aging after removal of it are clearly seen. The decay of piezoelectric response after poling exhibits an initial fast component which reflects the back switching of previously aligned domains and a slow decrease which may be fitted by the equation

$$
d_{33}=A-B \log (t)
$$

Such a logarithmic decay has been frequently observed in aging studies of bulk ferroelectric materials and was attributed to a broad distribution of the relaxation times of species involved in aging process $[8,9]$.

The aging rates and values of piezoelectric coefficients after poling were found to be sensitive to poling time. The longer the poling time, the more stable domain configuration is created and the lower is the aging rate (Fig. 2). Practically, a poling time of about several tens of minutes is sufficient for the saturation of aging rates (Fig. 3). This time is close to the Maxwell relaxation time in these films and may correspond to the fixation of the existing domain configurations by space charges.

It is important to estimate the life time of piezoelectric device subjected to poling. Extrapolation of the data shown in Fig. 2 to longer times yields a 25\% decrease of the initial response after about 2 years.

The aging rate was also found to depend on the direction of the poling field. For these measurements, virgin films were used without any deaging procedure. The films which exhibited no preferred direction of polarization (the piezoelectric coefficient was small for virgin films) showed similar aging rates when positive or negative voltages were used for poling. However, in films with a distinct preferred direction of polarization (the initial piezoelectric response is close to that after poling), the aging rates were different, being higher when the poling field was applied against the preferred polarization direction (Fig.4). The strong internal field which is created by non-homogeneous charge distribution inside the film [10] promotes the fast destabilization of the domain structure when the positive voltage is used for poling.

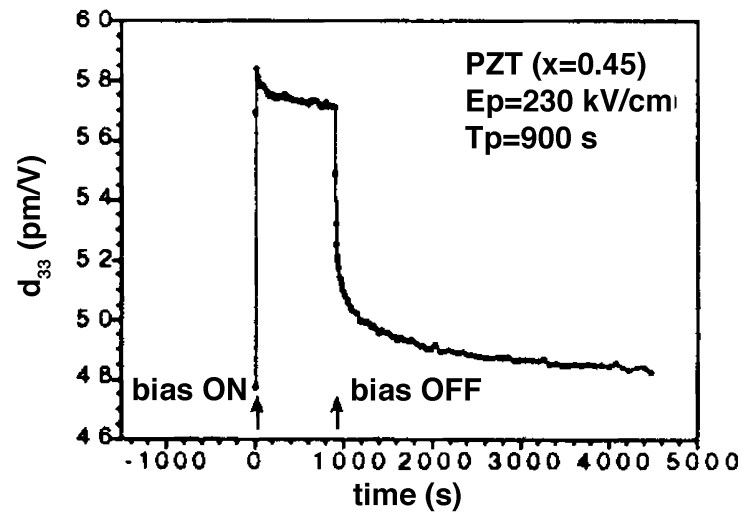

Figure 1. Typical piezoelectric response of PZT $(x=0.45)$ film under de bias and after poling.

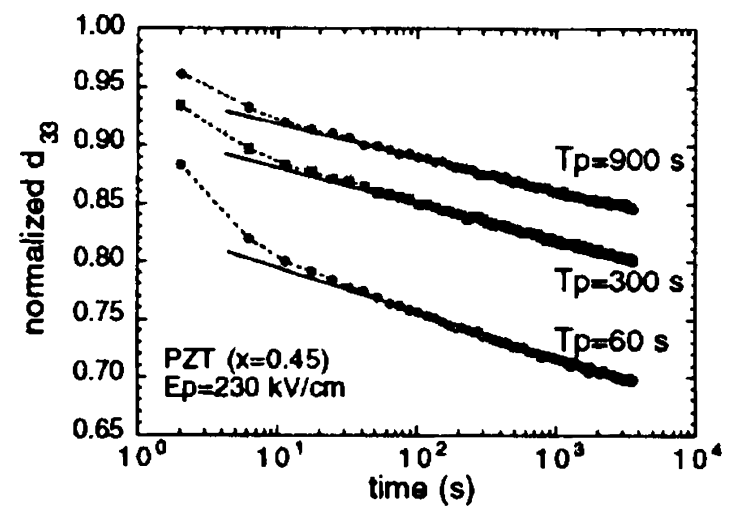

Figure 2. Aging curves for PZT $(x=0.45)$ films poled with different times. $d_{33}$ is normalized to its value under de bias. 


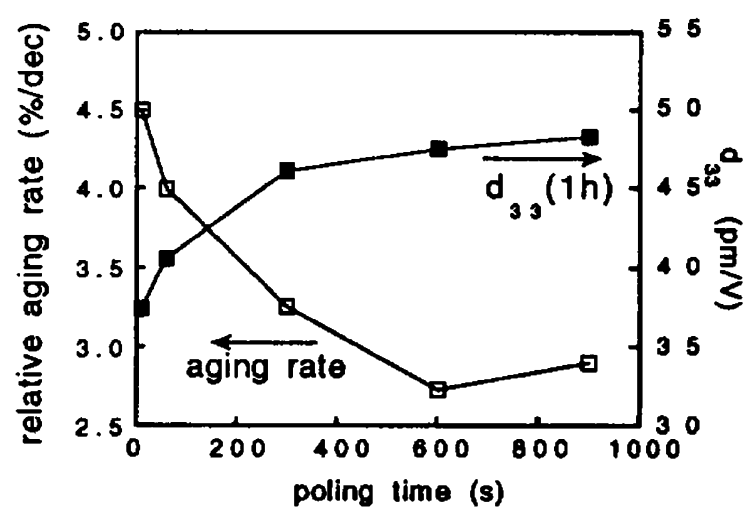

Figure 3. Aging rates and values of the piezoelectric coefficient at $\mathrm{t}=1 \mathrm{~h}$ after poling as a function of poling time for PZT $(x=0.45)$ films.

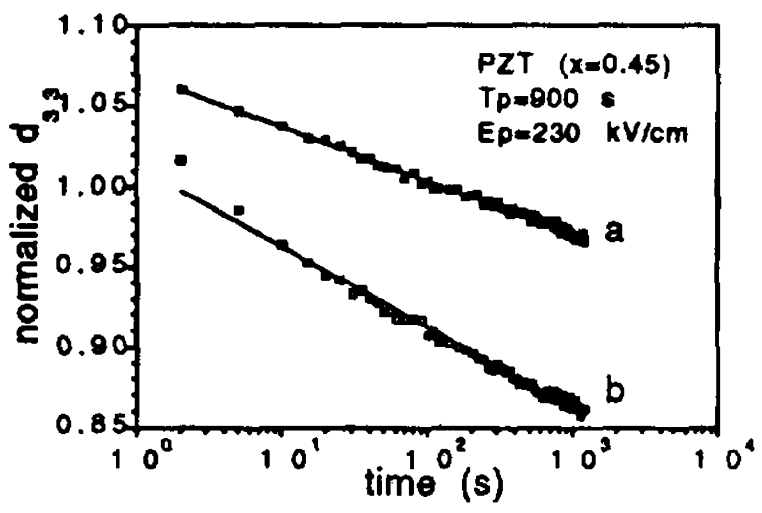

Figure 4. Aging curves after poling along (a) and against (b) the direction of preferred polarization. $d_{33}$ is normalized to its value under de bias.

The aging process in bulk ceramics is frequently accompanied by a translation of the polarization hysteresis loops in the direction opposite to that used for poling [9,11]. This translation was attributed to the domain stabilization and was described as the build-in of the internal bias field $[12,13]$. It is questionable if this internal field is a characteristic of thin films too $[14,15]$. Therefore, the measurements of piezoelectric hysteresis loops in the initial state and just after the end of aging process were performed. Only small changes in the coercive fields $(10 \mathrm{kV} / \mathrm{cm})$ which are close to those found in [14] could be seen. However, these small shifts can not confirm the conclusions about the negligible internal field effects in PZT films [14] because the piezoelectric hysteresis loop measurements are, in principle, destructive and the initial state may be restored by the prolonged application of dc bias [10].

It should be noted that aging rates of piezoelectric coefficient observed in this work are close to those reported previously in the aging studies of switching polarization of PZT films [14,15]. This means that piezoelectric aging is probably correlated with the aging of remanent polarization. Further measurements are needed to confirm this assumption.

\subsection{Fatigue}

Fatigue measurements under bipolar and unipolar cycling were done to elucidate the nature of the fatigued state and to evaluate the reliability of piezoelectric PZT films working in on-off mode. It is shown that bipolar cycling causes not only a strong decrease of piezoelectric coefficient after $10^{4}-10^{5}$ pulses which reflects the loss of switchable polarization, but also a shift of piezoelectric hysteresis loops which is considered to originate from locking of the domains in the preferable orientations [16].

For piezoelectric applications the behavior of piezoelectric response under unipolar cycling is of more importance because the films will never switch under the normal operating conditions. It was found that the degradation in this case is much smaller than that under the bipolar conditions. Again, if the voltage pulses are applied against the direction of preferred polarization the fatigue is more pronounced than in the opposite case (Fig.5). Simultaneously the symmetrization of coercive fields was observed. Obviously, the voltage pulses which act against the preferred polarization direction may switch more domains which become immobile by e.g. pinning of the domain walls by defects. This should decrease the contribution of the domain wall motion to piezoelectric response. 


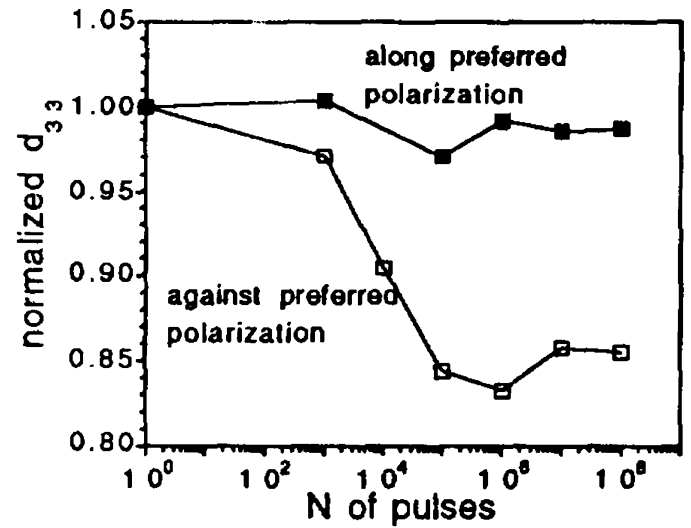

Figure 5. Normalized piezoelectric coefficient at $E=230 \mathrm{kV} / \mathrm{cm}$ as a function of number of voltage pulses $(300 \mathrm{KV} / \mathrm{cm})$ applied along and against the direction of preferred polarization.

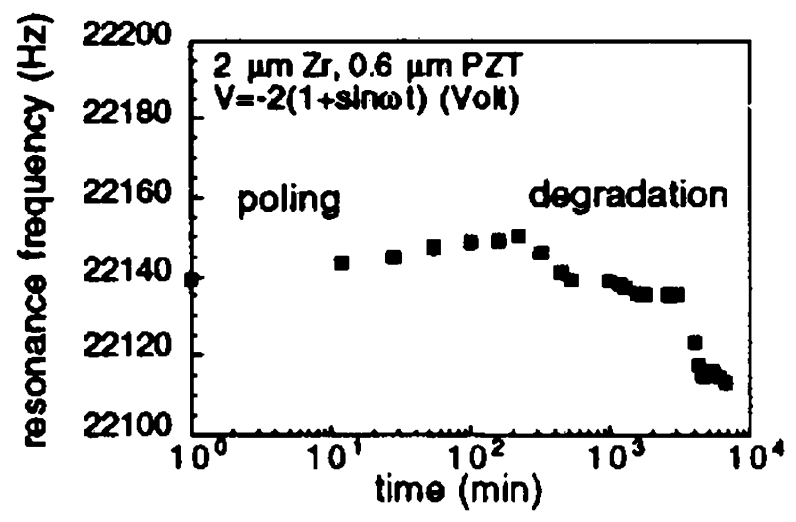

Figure 6. Resonance frequency of a $\mathrm{Zr}$ membrane covered by $0.6 \mu \mathrm{m}$ PZT film showing degradation under the conditions imitating the work of a piezoelectric ultrasonic motor.

\subsection{Degradation in piezoelectric devices}

The degradation of the piezoelectric coefficient described in previous sections should influence the reliability of piezoelectric devices. However, the degradation behavior will strongly depend on the type of the device as well as on the mode of its operation. Both degradation of the piezoelectric properties themselves and mechanical degradation of the devices (e.g. microcracking) should be taken into account. As an example, the degradation behavior of thin membranes covered by PZT film has been studied. The PZT film, through the transverse piezoelectric coefficient d3,' causes the vibrations in membrane which can be easily measured by the interferometer. The resonance frequency of the vibration is a good indicator of degradation because it depends not only on the mechanical properties of the membrane but also on the dc stress produced by the piezoelectric effect in the PZT film. The resonance frequency of Si membranes was found to increase with the increase of $d$,, under poling [17]. The behavior of resonance frequency with time under conditions which imitate the work of a piezoelectric ultrasonic motor [3] is shown in Fig. 6. The resonance frequency initially increases. This can be understood as a prolonged poling under the average dc field which is less than the coercive one. After some stabilization period, the resonance frequency starts to decrease, indicating a possible decrease of the piezoelectric coefficient with time. Finally, a drop of the resonance frequency is observed which is probably related to the change of mechanical properties of the $\mathrm{Zr}$ membrane.

\section{Conclusions}

In this work, several types of piezoelectric degradation in PZT films have been observed such as aging, fatigue and degradation under the dc bias. All of them result in deterioration of piezoelectric properties with time and may, in principle, limit the work of piezoelectric devices.

The poling time as well as the mutual orientation of the poling field with respect to the direction of preferred polarization are shown to be the main factors which determine the aging rates of piezoelectric coefficient. The aging rates observed in this study are consistent with those reported previously for switching polarization.

The bipolar fatigue results in the strong decrease of piezoelectric coefficient which reflects the loss of switching polarization. Under unipolar conditions the fatigue is much less pronounced and may depend on the direction of applied field.

The degradation behavior of thin membranes covered by PZT films is explained by both the changes of the piezoelectric properties of PZT films and the mechanical properties of membranes with time. 


\section{Acknowledgments}

This work was supported by the European COST 514 Program and by the Materials Priority Program of the Board of the Swiss Federal Institute of Technology.

The authors gratefully acknowledge A. Tagantsev for helpful discussions and J. Llambias and C. Wüthrich for their invaluable assistance during the construction of laser interferometer.

\section{References}

1. D. L. Polla, C. Ye, P. Schiller, T. Tamagawa, W. P. Robbins, D. Glumar, and C. C. Hsueh, Mat. Res. Soc. Symp. Proc., 243 (1992) 55.

2. P. Schiller and D. L. Polla, Proc. 7th Conf. On Solid State Sensors and Actuators, (1993) 154.

3. P. Muralt, M. Kohli, T. Maeder, A. Kholkin, K. Brooks, N. Setter, and R. Luthier, Sensors and Acuators A, 48, (1995) 157.

4. K. D. Budd, S. K. Dey, and D. A. Payne, Brit. Ceram. Proc., 36 (1985) 107.

5. K. Brooks, D. Damjanovic, A. Kholkin, I. Reaney, N. Setter, P. Luginbuhl, G. A. Racine, N. F. de Rooji, and A. A. Saaman, Integrated Ferroelectrics, 8 (1995) 13.

6. W. Y. Pan and L. B. Cross, Rev. Sci. InsIr., 60 (1989) 2701.

7. Q. M. Zhang. W. Y. Pan, and L. B. Cross, J. Appl. Phys., 63 (1988) 2492

8. K. W. Plessner, Proc. Phys. Soc. (London), 69 (1956) 1261.

9. W. A. Schulze and K. Ogino, Ferroelectrics, 87 (1988) 361.

10. G. E. Pike, W. L. Warren, D. Dimos, B. A Tuttle, R. Ramesh, J. Lee, V. G. Keramidas and J. T. Evans, Appl. Phys. Lett., 66 (1995) 484.

11. P. V. Lambeck and G. H. Jonker, J. Phys. Chem. Sol., 47 (1986) 453.

12. K Carl and K. H. Hardtl, Ferroelectrics, 17 (1978) 473.

13. U. Robels and G. Arlt, J. Appl. Phys., 73 (1993) 3454.

14. W. H. Shepherd, Mat. Res. Soc. Symp. Proc., 200 (1990) 277.

15. R. D. Pugh, M. J. Sabochick. and T. E. Luke, J. Appl. Phys., 72 (1992) 1049.

16. E. L. Colla, A. L. Kholkin, A. K. Tagantsev, K. G. Brooks, and N. Setter. this issue.

17. P. Muralt, M. Kohli, T. Maeder, K. Brooks, A Kholkin, R. Luthier, and N. Setter, this issue. 